\title{
A FRAMEWORK FOR MANAGEMENT OF VIRTUAL ORGANIZATION BREEDING ENVIRONMENTS
}

\author{
Hamideh Afsarmanesh ${ }^{1}$, Luis M. Camarinha-Matos ${ }^{2}$ \\ ${ }^{1}$ University of Amsterdam,THE NETHERLANDS, hamideh@science.uva.nl \\ ${ }^{2}$ New University of Lisbon / Uninova, PORTUGAL, cam@uninova.pt
}

\begin{abstract}
Effective creation of dynamic virtual organizations requires a proper breeding environment to increase organizations' preparedness. After introducing some basic concepts related to collaborative networked organizations, the concept of breeding environment for virtual organizations is discussed and the key elements and requirements for its support management system are presented. The initial modeling needs and the required functionality are identified. Finally some important open challenges are addressed.
\end{abstract}

\section{INTRODUCTION}

Collaboration among autonomous and geographically disperse entities is a process that is clearly facilitated by the advances in computer networks and related technologies. A growing number of collaboration networks, including the "classical" virtual enterprises [11],[3], virtual organizations [10], and professional virtual communities [1],[6], as well as new organizational forms [2], are emerging. As a result of intense $R \& D$ in this area, new operating and governance rules as well as support environments are being developed.

Dynamic collaborative networks able to rapidly form and adapt to changing conditions provide good intuitive approaches to face the challenges of turbulent markets [10]. A key question is however how to guarantee the basic requirements to enable such collaboration. Among others, the formation of any collaborative coalition depends on its members sharing some common (or compatible) goals, possessing some level of mutual trust, having established common (interoperable) infrastructures, and having agreed on some common (business) practices and values. Achieving these conditions is a pre-requisite for agility and integratability in collaborative networks.

One discussion point is whether such organizations shall be temporary or longterm establishments. Temporary organizations seem to better fit the dynamics of the market and the typically short duration of business opportunities, while long-term organizations better cope with the trust building process and the investment on common infrastructures and practices. Traditional supply chains and some forms of extended enterprises in stable mass-production oriented businesses are examples of long-term organizations. Some interesting experiments combine both types of organizations: A long-term club of organizations that are prepared for cooperation and the short-term coalitions involving different subsets of these organizations that are dynamically assembled in order to respond to business opportunities. These new organizational forms are early manifestations of breeding environments for dynamic 
virtual organizations and emerge as an evolution of the industry clusters and industrial districts [9], [4], [12], [13].

This paper discusses this combined approach in detail, identifying its components and requirements, and proposing the necessary modeling and functional requirements for a supporting management system as well as extending it with further facilitating component and services. This research is being developed within the framework of the IST Integrated Project ECOLEAD which aims at creating necessary foundations and mechanisms for establishing an advanced collaborative and network-based industry society in Europe.

\section{BASE CONCEPTS}

Early works have assumed that partners for a new VO could be easily identified and selected from the wide open universe of available enterprises / organizations, and merged into a collaboration network. This assumption however overlooks a number of important obstacles in this process among which the following can be mentioned: How to know about the mere existence of potential partners in the open universe and deal with incompatible sources of information? How to acquire basic profile information about organizations, when there is no common template or standard format? How to quickly establish an inter-operable collaboration infrastructure, given the heterogeneity of organizations at multi-levels, and the diversity of their interaction systems? How to build trust among organizations, which is the base for any collaboration? How to develop and agree on the common principles of sharing and working together? How to quickly define the agreements on the roles and responsibilities of each partner, to reflect sharing of tasks, the rights on the produced results, etc.?

As a basic rule, in order to support rapid formation of collaborative networks, e.g. a business consortium, it is necessary that potential partners are ready and prepared to participate in such collaboration. This readiness includes common interoperable infrastructure, common operating rules, and common cooperation agreement, among others. Any collaboration also requires a base level of trust among the organizations. Therefore, the concept of breeding environment has emerged as the necessary context for the effective creation of dynamic virtual organizations. We define the Virtual organization Breeding Environment (VBE) [9] as:

an association of organizations and their related supporting institutions, adhering to a base long term cooperation agreement, and adoption of common operating principles and infrastructures, with the main goal of increasing both their chances and their preparedness towards collaboration in potential Virtual Organizations.

A main general aim of the VBE is focused on the transition from point-to-point connections among organizations to a network structure, in order to increase the chances of its member organization's involvement in opportunities for collaboration. Traditionally, breeding environments are established within one geographic region, in the tradition of industry districts, with the advantage of having common business culture and sense of community, as well as focusing on one specialty sector of the region. But, this restriction can today in most cases be overcome by VBEs. 
VBEs primarily constitute two categories of regional and global. While regional VBEs mainly involve organizations (of different sizes) from one geographical region, a global VBE incorporates the involvement of geographically distributed organizations. In this paper, we address global VBEs. Furthermore, both regional and global VBEs can be either single-sector, i.e. specializing in a single focus area, or multi-sector, i.e. covering a number of focus areas.

Efficient creation of VOs is the main purpose for the existence of the VBEs. Therefore, the motivation for creation of VBEs primarily depends on identification / creation of opportunities for organization collaborations in certain sector(s). There are two kinds of opportunities pursued by a VBE, namely those that can be identified in the market/society, and those that can be created by the VBE for the purpose of innovation. The main actors in creation/identification of opportunity are either the VBE members who broker the VOs, or the VBE administrator who promotes the initiation of some VOs that seem to be beneficial for the market/society.

Establishment of VBEs provides the ten advantages listed and exemplified below, that are further described in the paper:

1. Agility in opportunity-based VO creation

- Supporting reduction of needed efforts and complexity, flexibility for VO re-configurability, and cost effectiveness

2. Provision of base effective IC technology infrastructures for VBE members

- The common grounds for interoperability / inheritability / collaboration

3. The VBE bag of assets, providing properties of interest for its members

- General sharable information/knowledge (e.g. standardized product definitions and processes), software tools, lessons learned

4. Provision of mechanisms, guidelines, and assisting services to both motivate and facilitate configuration and establishment of VOs

- Creating system of incentives, mechanisms to create positive reputation, and services for partners search, contract negotiation, etc.

5. Proactive management of competencies and resources available in VBE

- Assuring coverage of the needed competency/resources within the VBE

6. Provision of related consulting/life maintenance support for VBE members through its support institutions

- Supporting insurance, branding, training, etc.

7. Introduction of approaches/mechanisms to build trust among VBE members

- By recording the performance history, and definition of criteria for organizations' trust worthiness

8. Provision of general guidelines for collaboration

- Constituting rules of conducts, working and sharing principles, value systems, collaboration ethics and culture, IPR protection, etc.

9. Increasing the chances of VO involvement for VBE members, even from remote geographic regions

- Through provision of members' profile in the VBE catalog, including their competencies, resources, products, services, etc.

10. Improving the potential / capacity of risk taking by the VO initiators

- Due to the reduction of the VO setup efforts/time, availability of both a wide variety of competency/resources as well as indicators of the level of trust worthiness and past performance of the VBE members 
A VBE is a regulated open, but controlled-border association of its members. It aims at improving the preparedness of its member organizations for joining potential future VOs, hence providing a cradle for dynamic and agile establishment of opportunity-driven collaborative networks. As represented in Fig. 1, it is far less costly and much more effective to quickly build a VO in a breeding environment context (branch 1b) than through a generalized partners' search (branch 2). In other words, VBEs substantially contribute to the increase of the level of preparedness of their members for participation in potential collaborative processes.

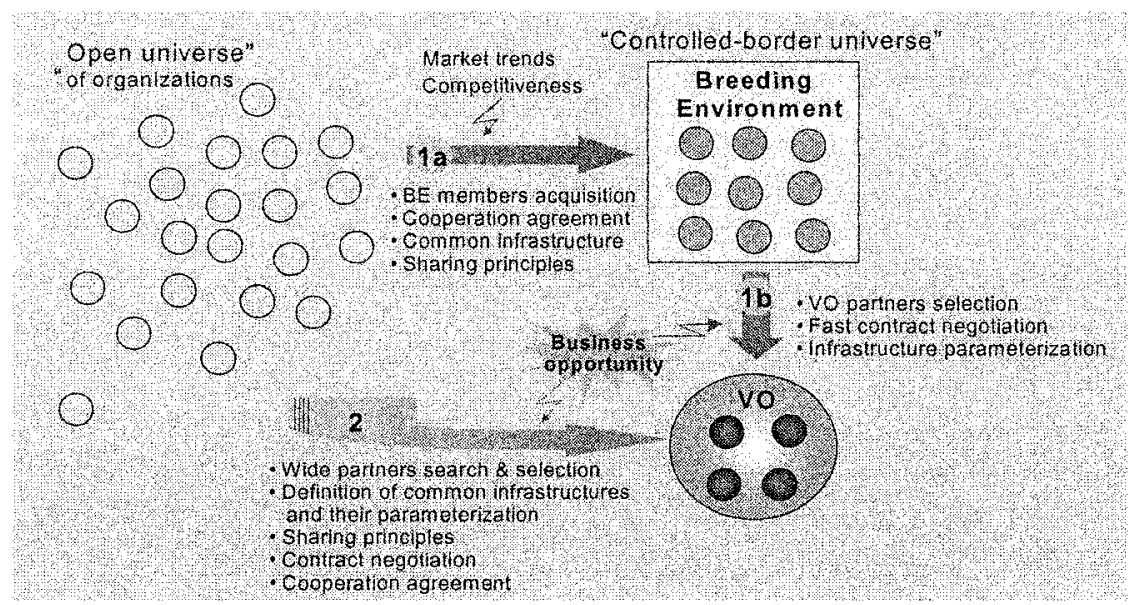

Figure 1 - Two approaches to the formation of virtual organizations

A VBE must have a controlled boarder, but does not need to be closed boarder; namely at any time new members can join the VBE association by complying with its general operating principles. Therefore, there may be different levels of membership defined and supported by the VBE administrator, each corresponding to different set of rights and responsibilities. In principle, different levels of VBE membership may constitute a range, with loose-membership on one end and tightmembership on the other end of the spectrum. For instance, a loosely associated member of the VBE may need to adhere to nothing more than a minimum level of organization "preparedness" that is necessary for getting involved in a VO, and to making some minimum information available to the VBE administration, e.g. about their activities related to the VO. At the same time, typically a fully active member of the VBE contributes to its promotion, growth, and the enrichment of its bag of assets, and can take an active role from brokerage and planning of VOs in a niche market, to being involved in the expansion of the VBE into new sectors, and initiating VOs towards innovation.

Similarly, for the formation of a VO that is initiated within the VBE, while preference will be given to the VBE members, it might be necessary to find some external organizations, for example when some skills or capacities are not available inside the VBE. Nevertheless, before becoming a partner in such a VO, an external organization shall be invited to join the VBE, at least at the loosest level (e.g. as a loosely associate member), in order to cover its minimum necessary VBE compliance. In some cases, it may be even desirable (either by organizations or by the VBE administration) that the names of the restricted members (e.g. the loosely 
associated members) of the VBE are not publicized as the VBE members. For instance if the association of an external organization with the VBE is only due to the fact that they are currently involved in one running VO initiated by the VBE, and this membership will not continue after the VO dissolves.

In addition to the enterprises, a VBE might include other kinds of organizations (such as consulting/research institutes, sector-associations, governmental support organizations, etc.) and even free-lancer individual workers that represent a oneperson small organization. Furthermore, VBE can include and serve as the hosting environment for some support-institutions that will provide some specialized related services/expertise to the VBE members, such as the legal services, marketing expertise, etc. for the VOs configured within the VBEs, or the insurance services, training support, etc. (also called "life maintenance" support) for the free-lancers involved in the VBE.

Members of the VBE are the organizations that are registered at the VBE (traditionally bound to a sector). In summary, organizations in VBE represent:

- Business entities providing products and services to the market that get involved in the VOs to gain quantitative profit.

- Non-profit institutions that get involved in the VOs to gain qualitative profit.

- VO Support institutions, for example: legal and contractual service providers, companies supporting life maintenance to individuals (e.g. insurance and training companies), ministries, sector associations, chamber of commerce, environmental organizations, etc.

VBE members must comply with the general VBE rules and policies, e.g. adopting the common ICT infrastructure. At the same time, once joined the VBE, member organizations have access and shall benefit from the following available elements among others: Common information, services, and tools constituting its bag of assets; Common market and distribution channels; Common resource and labor pool; Common VBE cultural ties; Facilities to share the cost of new experiences; Facilities to share lessons learned.

$A$ variety of roles can be assumed by a large number of actors in the VBE, where an actor represents either a VBE member organization or an individual representing a VBE member organization [9],[10]. Due to the autonomous nature of the VBE member organizations, at different times (or even simultaneously) the same organization may assume different roles, e.g. acting as a VO participant, a VO coordinator, etc. where each role requires assigning different rights/responsibilities within the VBE. Supporting these actors with their roles is a high priority in the VBE, and providing needed information and assisting tools for their support is among the VBE environment challenges. The following main roles are considered for the VBEs:

- VBE Member - this is the basic role played by those organizations that are registered at the VBE and are ready to participate in the VBE activities.

- VBE Administrator - performed by the organization responsible for the VBE operation and evolution, promotion of cooperation among the VBE members, filling the skill/competency gaps in the VBE by searching and recruiting/inviting new organizations into the VBE, daily management of the VBE general processes, e.g. assignment/reassignment of rights to different actors in the VBE based in their responsibilities, conflict resolution, preparation of a bag of VBE assets, and making common VBE policies, among others. 
- Opportunity Broker or simply Broker - performed by a VBE actor (a VBE member organization or an individual representing a VBE member) that identifies and acquires new collaboration opportunities (of business nature or others), by marketing VBE competencies and assets and negotiating with potential customers. There is also the possibility of this brokerage function being played by an entity outside the VBE as a service to the VBE.

- VO Planner or business integrator - performed by a VBE actor that, in face of a new collaboration opportunity (designed by an opportunity broker), identifies the necessary competencies and capacities, selects an appropriate set of partners, and structures the new VO. In many cases the roles of opportunity Broker and VO planer are performed by the same actor.

- VO Coordinator - performed by a VBE actor that will coordinate a VO during its life cycle in order to fulfill the goals set for the collaboration opportunity that triggered the VO.

A number of other roles might be also useful to be considered in a VBE, including: the VBE Advisor (or an advisory board), the VBE Service Provider, the VBE Ontology Provider, the VBE Expertise Provider (through a support institution) involved in the VBE, and the last but not least is the role of VBE Guest played by an organization outside the VBE that is interested in finding general promotion information about the VBE, either interested to become a VBE member or interested in contacting the VBE in relation to a business opportunity, etc.

Because of the dynamic nature of both the VBE's environment and its member organizations, the defined roles for member organizations cannot be static. Every role taken by a VBE member organization represents: a set of responsibilities, a set of required rights/authorization, and further requires a set of assisting tools for the actor in that role. For instance, a VBE member, acting in the role of a VO broker, has accepted the responsibility to configure and negotiate a VO, for which it requires a set of access/visibility rights to the information regarding competency/pastperformance of other member organizations available in the VBE, and requires an assisting tool to search for best fit organizations matching the required skills.

Considering the rights that need to be associated with every role of an actor in the VBE, it is necessary that the VBE members inform the VBE administrator about every new role they plan to assume (starting with becoming a VBE member organization) in order to request for proper rights to be associated to them.

The life cycle of the VBE represents all the stages that a VBE may go through, from its creation stage, to its operation, and possible dissolution (see Fig. 2 for VBE life cycle stages). In fact VBE, being a long-term alliance, and considering its valuable bag of assets gradually collected in the VBE, its dissolution is a very unusual situation. Instead, it is much more probable that the VBE goes through another stage, that we call the metamorphosis stage, where it can evolve by changing its form and purpose. The creation stage can be divided into two phases, namely (i) initiation and recruiting, dealing with the strategic planning and initial incubation of the VBE; (ii) foundation, dealing with the constitution and start up. The VBE creation needs to be properly supported considering the increasing variety of VOs, and the fact that usually every VBE serves a specific sector/domain and has specific aims. In the coming time, there will be a large number of different sector/domain-dependent VBEs needed to be established, in order to better support the creation of different forthcoming VOs. The VBE life cycle stages, as seen in 
Figure 2, include: the VBE creation - composed of the VBE initiation/recruiting and the VBE foundation, the VBE operation/evolution, the VBE metamorphosis, and the VBE dissolution.

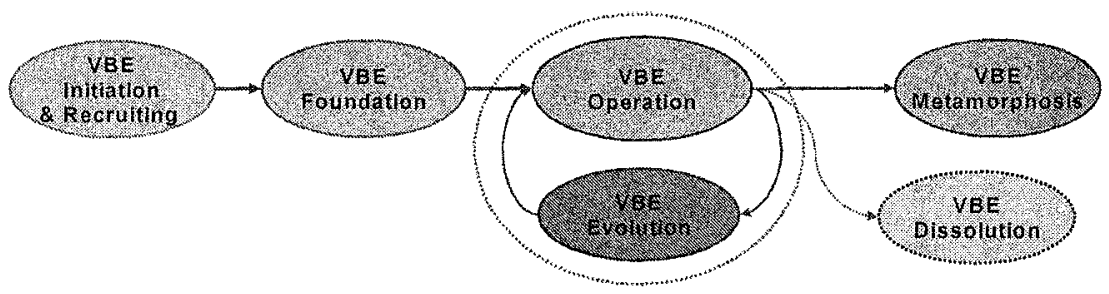

\begin{abstract}
VBE Initiation \& Recruiting - planning and incubation
VBE Foundation - constitution and start up

VBE Operation - the "normal" phase of the VBE existence

VBE Evolution - small changes in membership and daily operating principles

VBE Metamorphosis - major changes in objectives, princlples, membership

and/or mergers, leading to a new form of organization

VBE Dissolution - when the collaborative entity ceases to exist;

Being a long-term entity, this stage of VBE is typically replaced

by the metamorphosis stage, preserving the gained knowledge.
\end{abstract}

Figure 2 - VBE life cycle stages

Table 1 shows some effective examples of traditional breeding environments that provide substantial evidence for the need to establish VBE frameworks and the necessity of developing VBE support functionalities.

Table 1 - Examples and potentials of VBEs

\begin{tabular}{|l|l|l|l|}
\hline Case & Menuers & Tocation & Domain \\
\hline Virtuelle Fabrik & 100 & $\begin{array}{l}\text { Switzerland, } \\
\text { Germany }\end{array}$ & Mechanical industry \\
\hline Kiesel & $>15$ & Germany & Services, Environment \\
\hline Virtec & $>9$ & Brazil & Manufacturing \\
\hline CEFAMOL & 136 & Portugal & Plastic moulds \\
\hline $\begin{array}{l}\text { Virtual Enterprise } \\
\text { Networks Yorkshire }\end{array}$ & $>25$ & UK & $\begin{array}{l}\text { IT, Machinery, Bio-tech, e- } \\
\text { Learning }\end{array}$ \\
\hline Bipolo Ticino & $>13$ & Switzerland & Life sciences \\
\hline Virtual Biotech Company & $>150$ & Germany & Biotechnology \\
\hline PVC & 45 & Australia & Plastics \\
\hline Regional Net for Ontario & - & Canada & Telecommunications \\
\hline VIRFERBRAS & $>12$ & Brazil & Moulds \\
\hline Fenix Cluster & $>250$ & Mexico & Electronics, metal \& plastic \\
\hline Biotechnology cluster & 411 & USA & Biotechnology \\
\hline Biotechnology cluster & $>160$ & Canada & Agro-food, biotechnology \\
\hline $\begin{array}{l}\text { Advanced Business } \\
\text { Services }\end{array}$ & $>6$ & USA & Credit, lending, investments \\
\hline Helsinki ICT cluster & 79 & Finland & Telecommunications \\
\hline CARPI & 2068 & Italy & Textile/ clothing \\
\hline Mining Cluster & - & Chile & Mining industry \\
\hline Motorsport Valley & 40 & UK & Motor-sport \\
\hline Verkko A & 12 & Finland & Process industry \\
\hline Automotive cluster & 54 & Slovenia & Automotive industry \\
\hline Plasttechnics cluster & $>60$ & Slovenia & Plastics \\
\hline
\end{tabular}




\section{MODELING NEEDS}

In order to design and develop a management system for VBEs it is necessary to first identify and model the main elements of this collaboration environment. A wide variety of entities and concepts co-exist in a typical VBE environment, as graphically represented in Fig. 3. VBEs include heterogeneous organizations of

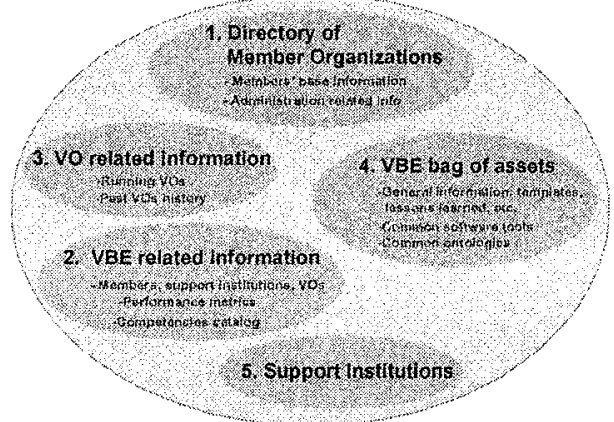

Figure 3 - Main categories of information in a VBE management system different sizes and a number of support institutions. The VOs are from time to time created and will interact with the VBE. Furthermore, the VBE management system controls the base running environment enforced by a number of policies and regulations, as well as providing a set of general common tools, facilities and information that constitute the bag of assets for the VBE. Therefore, a large variety of information and knowledge must be properly modeled, organized, and applied to manage the VBE.

For most of the elements in the categories identified in Fig. 2, generic sectordependent/application-independent specifications can be provided, namely a common ontology can be defined for those categories and included in the VBE management system, to be used for all sectors and applications. However, some of the identified elements in Fig. 2 cannot have a common sectordependent/application-independent definition. These elements include the competencies, resources, products and services of the VBE, for which their modeling and ontology need to be handled differently, as addressed below.

VBE Products and services specification is widely varied among different sectors. Furthermore, different products or services have different specifications and their respective properties describing each product or service differ widely, and thus their definition/conceptualization (ontology) will be completely different as well. In the best case, if there are standard meta-data / ontology defined for a specific sector for which the VBE is established, these standard models can be stored within the VBE as a part of its general assisting information, in its bag of assets. Otherwise, an alternative approach is needed to generate/define their ontology. For this purpose, some text mining tools can be developed to help discover ontology-related concepts and keywords. The approach planned for the ECOLEAD, semi-automatic mechanisms will be developed to mine and derive concepts from the online corpus related to VBE member organizations, in order to present to the human experts in the sector, assisting them with gradual / incremental building up of such meta-data and ontology for the VBE.

Similarly, competencies and resources offered by the VBE are also not easy to generalize. Consider for instance the differences in competency definitions (metadata/ontology) that describe the skills / knowledge / potential of the organizations related to examples provided in Table 1. Furthermore, consider and notice the wide variety and heterogeneity among resources owned, such as facilities/machineries related to different domain/sectors. Clearly neither Competencies nor resources of 
the organizations in the VBE for Healthcare (e.g. hospitals, care centers, Doctors' practice office, insurance companies, ambulance services, etc.) can be defined by the same ontology (specification) that defines these aspects of the organizations in the VBE for production and assembly of bicycles (e.g. the raw material provider, mould maker, paint manufacturer, factory, marketing firm, packaging company, etc.).

Therefore, in the above case, only a minimum common meta-data can perhaps be defined and modeled, as the starting point for defining the competency and resources meta-data. This common meta-data includes a generic upper ontology plus some minimum profiling information common to all potential VBE member organizations. From that point on, similar to the product/services, some semiautomatic mechanisms need to be applied to VBE to continuously support the derivation/discovery of its domain/sector meta-data, and incrementally build and expand some common ontology for the competencies (and resources) of the organizations involved in that sector. For instance, semi-automatic mining of on-line texts, either directly through questionnaires and brochures provided by the current VBE members or through their web sites, can discover common elements of the competency related to the VBE sectors.

Nevertheless, it is important to notice that for proper modeling of the VBEs information, the development of a strong and detailed ontology for organizations' competency (related to the sector represented in the VBE), as well as the proactive management of this competency catalog are significant. This is due to the need to assist the opportunity brokers and to increase the effectiveness of the search/matchmaking process, necessary for creation of VOs within the VBE.

\section{LIFE CYCLE SUPPORT FUNCTIONALITY}

Defining a comprehensive and generic "reference model" for VBEs is a big challenge. Nevertheless, based on the initial empirical knowledge gathered from existing cases (see examples in Table 1), it is realistic to design the first steps for gradual definition of a "reference framework for VBEs", addressing aspects such as the VBE behavior, topology, and structure.

In this direction, in addition to identification of constituting entities and concepts in VBEs that are briefly addressed earlier in the paper, so far a first list of required functionality for a VBE management system is identified in the ECOLEAD in relation to different stages of its life cycle. A subset of this list is addressed below and partially represented in Fig. 4.

Base functionality supporting the VBE creation - This phase includes two main steps: (1) initiation / recruiting, which requires the establishment and setup of a common base ICT infrastructure, and establish some base ontology / thesaurus of the domain, once the vision and strategic objectives of the VBE are defined; (2) $V B E$ foundation, requiring support for parameterization of the used systems, setting up the necessary links, recruiting potential organizations to join the VBE (founding members), creation of the necessary databases (with initial meta-data / ontology), and populating these information structures. 


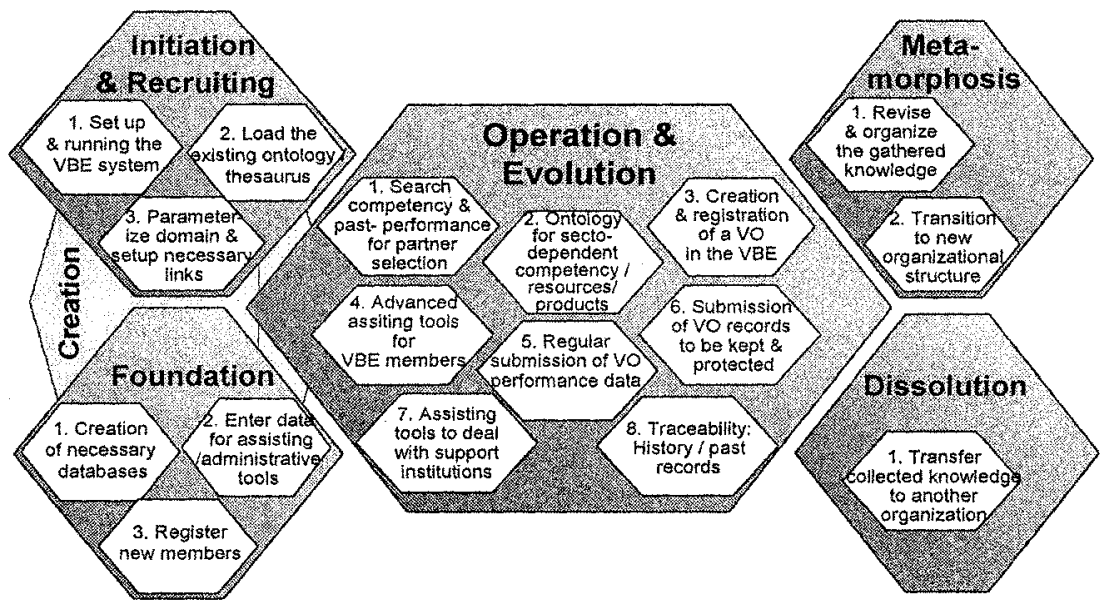

Figure 4-Examples of VBE life cycle support functionalities

Base functionality supporting the VBE operation and evolution - This phase requires support for: management of competencies and assets, registration of new members (including incremental profiling, characterization of competencies, products, services, etc.), assisting VO creation, incremental generation / evolution of ontologies for the domain / sector, keeping records of past performance and collaboration processes, assessment and assistance tools, collaboration support (e.g. newsgroups, discussion forum, common information repositories, etc.), management and evolution of working and sharing principles and rules, acquisition and management of common knowledge and assets.

Base functionality supporting the VBE metamorphosis - This phase will require assistance for the design of the aimed new organizational structure, selection and reorganization of the information and knowledge collected during the VBE operation, and that might be transferred to the new organization, analysis and adjustment to the new context, etc.

Base functionality supporting the VBE dissolution - In the case of dissolution there is a need to plan the transfer of its collected knowledge, information, bag of assets, etc. to its members, or another entity based on defined agreements.

Clearly, considering the existing technological approaches, more than one system architecture can be designed for the VBEs, and many different ICT tools and mechanisms can be applied to develop this architecture [8].

\section{SOME OTHER CHALLENGES}

A number of important challenges can be identified in the design and development of a replicable VBE framework, where competency management, value systems, system of metrics, and trust management are among the main elements.

Competencies Management: Competencies represent the "capacity" for existing resources plus the available capabilities/skills to perform some task or activity. Competencies are thus the combination of capabilities and capacities. Proper management of the set of competencies is fundamental for a VBE management 
system. The VBE Competency Management System (CMS) shall include a catalog of competencies and a set of functionalities to manage it. In VBE, potential users of the CMS include the VBE Administrator, Broker, VO planner, VO Coordinator, and VBE Adviser. Table 2 summarizes some of the important issues needed to be considered in the CMS.

\section{Table 2. Issues in Competencies Management}

\section{Competencies catalog:}

- Ontology of competencies / skills - competencies need to be properly specified and organized in some taxonomic structure, supported by suitable navigational interface

- Related competencies/skills must be identified as means for replace-ability or equivalence of skills

- Some hierarchy of competencies must be defined and supported for search, for instance the more generalized (e.g. welding) as well as some specializations (e.g. spot welding).

- Furthermore, the inter-relationships (e.g. the IS-A, the whole-part, etc.) among competencies / skills must be defined.

- Properties aggregation to characterize each competency.

- Basic catalog entities manipulation - flexible search (muiti-criteria), add, remove, edit, etc.

- Internal Core and some non-core - For each member we can distinguish between the core and the non-core competencies.

- External valuation of VBE competency - Considering the global market level of the competency of the VBE as a whole.

- From an "operational" point of view, how to determine / collect competencies? Automatic? Manual? How to update them?

- Dynamic properties?

- It may be interesting to consider levels of competencies / skills. For a given competency C, does our VBE master it to the level of setting strategic developments, or is it only able to "follow" what is suggested by a customer, for instance.

\section{Competencies Management functionalities:}

- Competencies appraisal functionality - How "robust" or "sustainable" is the competencies base?

- Skills' gap analysis. What if one member leaves? Specific indicators of skill robustness? Skill robustness inside an organization - skill possessed by one employee or part of the "culture" of the organization? How to rate the skills base?

- Marketing support functionality - How to "sell" it to the outside?

- Identification of strengths and weaknesses? (Dynamic) Identification of new potentials?

- Acquisition of complementary skills? Which support functionality?

- Internal competencies \& external but "easily" accessible competencies

- What is the "unit"? Enterprise? Department? Group?

- Relationship to processes and roles

- Endorsement / "accreditation" of competencies. Or rating their quality/past performance?

- Notion of joint competency (when one specific competency results from the contribution of 2 or more partners, and such competency cannot be provided by a single partner). Or is this represented by the notion of complex competency (see above)?

- Others to be determined.

Value Systems. It is commonly accepted that the behavior of an individual, society, or ecosystem is determined by the underlying value system. It is intuitively understood that the values considered in a business-oriented VBE are different from those in a non-profit context (e.g. disaster rescue and incidence management network). Taking the simplified view that the goal of a VBE is to maximize some "attribute" of its value system, within a business context the dominant value is the profit (in economic sense), while in other cases the objectives are altruistic and compensated by the amount of prestige or social recognition, etc.

A value system is in essence the ordering and prioritization of a set of values that an actor or a society of actors holds. However, the values that a group or an actor holds may fall into several different categories since the concept of value is multifaceted. In order to define a value system for a VBE, we need to first define the 
characteristics of values that could be represented in a value system. A number of characteristics can be considered for the values of a value system:

- Subjective - For an identical context, distinct members may apply different values.

- Personal vs. social - The personal values can be applied only to one member (e.g. assets, capacity, and price) while social values can be applied to a set of members at VBE or VO level (e.g. ethical code, cooperation agreements, contracts).

- Normative - The purpose of normative values is to define a set of rules ("rights/duties") that contribute to assure the stability and the cohesion of the group since they transmit norms in a persuasive way and present an unquestionable form (e. g. trust, ethical code, and law).

- Exchange - The purpose of exchange values is to "measure" the objects exchanged among partners in a certain context and moment.

- Dynamic - The values can change along the time. They can fade out, increase their worth or can appear for the first time.

- Measurable - The values can be measured using a quantitative or a quantitative scale.

Definition of a value system in a VBE context is based on the notion that each product/service requires a set of value creating activities to be performed by a number of actors, forming a "value creating system" through a VO. As a result, value system needs to be defined at the VBE level, to provide:

- A regulation mechanism - for instance, to assure social cohesion, to understand members' behavior and to build performance indicators.

- A transactions mechanism between partners - such as, to assure an equality utility between objects exchanged.

For managerial purposes it is important to identify which values (within list of values) shall be part of the value system for each specific VBE (or application domain). Another related research challenge is the elaboration of some significant performance indicators - for VBE members, VOs, and the VBE as a whole - to be computed through some combination of values / variables of the adopted value system. Also, a related issue is the elaboration of procedures for distribution of profits resulted from some activities in the VBE (and VOs) [7].

System of incentives: The definition of a system of incentives is important for attracting and maintaining partners and members. In general, for business-related contexts, the key incentives to participate in a VBE are business benefits and knowledge. Incentives for members are mainly associated with costs, and some examples could be: (i) Guaranteed participation in a given number of VOs during a given period of time (although difficult to materialize in practice), (ii) A set of basic tools provided in the VBE bag of assets, (iii) On-demand VBE Product and Services, (iv) Tutorials, Courses and Conferences to enhance productivity (and core competencies) in companies, (v) An initial evaluation of the member and a commitment for constructive suggestions/advice to better its status in a given period of time.

The development of a set of performance indicators, as mentioned above, can complement these incentives by providing a more objective measure of the benefits of being involved in a VBE. As an incentive for member organizations to become 
more active in the VBE, a set of rules can be defined to collect points (e.g. a broker collects X points when

Incentives for government involvement are directly related to the social and economic impact of the VBE. Examples of this type of incentives are the increase in employment rates, increase in gross product, better infrastructures, and SMEs' development. For universities, the key incentives for participation in a VBE are the openness of VBE projects looking for student practices and an early introduction to industry practices. A better link between industry and academia can also be offered in order to improve research results. For R\&D organizations, the key incentive is the exploitation of their technological advances. This link between research and market is needed in order to sustain the research and development activities. Brokers are closely involved in materialization of this incentive.

Trust management. Trust is a critical antecedent for more efficient and effective communication, collaboration and knowledge creation. In fact innovation cannot be managed hierarchically because it depends on knowledge being offered voluntarily rather than on command. Therefore knowledge creation is social in nature; social exchange is a core process in knowledge creation.

Building trust is not an easy task, it requires mechanisms supported by the VBE, and usually takes time. Trust can be generated as an outcome of organization's past/present performance information recorded in the VBE. Therefore, trust is a component of the desired preparedness to participate in VOs. Trust plays an important role in order to create competitive advantage by reducing governance costs (management costs), costs for internalization (acquisitions), transaction costs between organizations, and impact positively in knowledge creation. Trust enables open communication, information sharing, and conflict management in a clear way, and also helps to speed up the contract process [5]. A set of well-established policies and criteria to follow in collaborative processes can help in creating / increasing trust among partners.

Several other challenges need to be also addressed including VBE marketing, ethical issues, management of VBE assets, contracts/collaboration agreements, etc.

\section{CONCLUSIONS}

For competing effectively in today's fast and rapidly changing business environment, organizations must organize people and processes to enhance responsiveness and flexibility and quickly react to a business opportunity. Advanced ICT and recently cutting edge web technologies have enabled new options in organizational structure and manifestations. Boundaries of location, time and individualization, and even organization have become less confining, allowing dramatic changes in the current work environments in form of VOs.

The Virtual organization breeding environment (VBE) is an emerging challenging area of research. Most elements comprising VBEs are not yet properly defined, and there are no reference models or reference architectures addressing the constituting elements and behavior of the VBEs. The multidisciplinarity of research on VBEs further adds to its complexity. Consequently, even discovery and identification of VBEs' requirements and proper definition of this problem area itself becomes challenging. Furthermore, to handle its wide variety of requirements, 
innovations in several areas are needed. Among others, endogenous to the VBEs, there are four key characteristics of VBEs that need careful attention. First, VBE entails the development of relationships with a broad range of potential partners, each having particular competency, resources, products, services, etc. that complements the others. Second, VBEs shall be regulated by a set of governing rules and principles for participation, and enforced by the VBE management system. Third, life cycle stages of VBEs determine its required functionality and services. And finally, there must be trust between VBE actors separated in space, for effective VO creation and operation. In this direction, ECOLEAD is contributing to the definition and establishment of a framework for VBEs that can be replicable to different application domains.

Acknowledgments. This work was supported in part by the ECOLEAD project funded by the European Commission. The authors thank the valuable contributions of their partners in the ECOLEAD consortium.

\section{REFERENCES}

[1] Afsarmanesh H., Guevara-Masis V., Hertzberger L.O., Virtual Community support in TeleCARE, in Processes and Foundations for Virtual Organizations, Kluwer, Oct 2003. [2] Afsarmanesh H., Kaletas E.C., Hertzberger L.O., The Potential of Grid, Virtual Laboratories and Virtual Organizations for Bio-sciences, in proceedings of 28th Conference on Current Trends in Theory and Practice of Informatics, SOFSEM 2001, Piest'any, Slovak Republic, 2001.

[3] Afsarmanesh H., Camarinha-Matos L.M. Future Smart-Organisations: a Virtual Tourism Enterprise. in proceedings of 1st International Conference on Web Information System Engineering - WISE'2000, Hong Kong, China, 2000.

[4] Bremer, C.; Mundim, A.; Michilini, F.; Siqueira, J.; Ortega, L. - A Brazilian case of VE coordination, in Infrastructures for Virtual Enterprises, Kluwer, Oct 1999.

[5] Blomvist, K. - Partnering in the dynamic Environment: The role of trust in asymmetric technology partnership formation, $\mathrm{PhD}$ thesis, Lappeenrant University of Technology, 2002.

[6] Camarinha-Matos L.M., Afsarmanesh H., TeleCARE: Collaborative virtual elderly care support communities, in The Journal on Information Technology in Healthcare, Vol.

2, Issue 2, London, Apr 2004, pp 73-86, ISSN 1479-649X.

[7] Camarinha-Matos, L.M.; Abreu, A. - A contribution to understand collaborative benefits, in Emerging solutions for future manufacturing systems, Springer, 2004.

[8] Camarinha-Matos, L.M.; Afsarmanesh, H. (Ed.s) - Collaborative networked organizations - A research agenda for emerging business models, Kluwer, 2004.

[9] Camarinha-Matos, L.M.; Afsarmanesh, H. - Elements of a base VE infrastructure, $J$. Computers in Industry, Vol. 51, Issue 2, Jun 2003, pp. 139-163.

[10] Camarinha-Matos, L.M.; Afsarmanesh, H.; Ollus, M. (Ed.s) - Virtual Organizations: Systems and Practices, Springer, 2005.

[11] Garita C., Afsarmanesh H., and Hertzberger L.O., The PRODNET Federated Information Management Approach for Virtual Enterprise Support. in Journal of Intelligent Manufacturing, vol. 12, pp. 151-170, 2001.

[12] Mejia, R.; Molina, A. - Virtual enterprise broker: Processes, methods and tools, in Collaborative business ecosystems and virtual enterprises, Kluwer, May 2002.

[13] Plüss, A.; Huber, C. - Virtuellefabric.CH - A source network for VE in mechatronics, in Virtual Organizations - Systems and Practices, Springer, 2005. 